\title{
Aerofoil broadband and tonal noise modelling using stochastic sound sources and incorporated large scale fluctuations
}

\author{
S. Proskurov ${ }^{\mathrm{a}}$, O. R. Darbyshire ${ }^{\mathrm{b}}$, S. A. Karabasov ${ }^{\mathrm{a}}$ \\ ${ }^{a}$ Queen Mary University of London, Mile End Road, London, E1 4NS, UK \\ ${ }^{\mathrm{b}}$ BAE Systems Computational Engineering, Filton, Bristol, BS34 7QW, UK
}

\begin{abstract}
The present work discusses modifications to the stochastic Fast Random Particle Mesh (FRPM) method featuring both tonal and broadband noise sources. The technique relies on the combination of incorporated vortexshedding resolved flow available from Unsteady Reynolds-Averaged NavierStokes (URANS) simulation with the fine-scale turbulence FRPM solution generated via the stochastic velocity fluctuations in the context of vortex sound theory. In contrast to the existing literature, our method encompasses a unified treatment for broadband and tonal acoustic noise sources at the source level, thus, accounting for linear source interference as well as possible non-linear source interaction effects. When sound sources are determined, for the sound propagation, Acoustic Perturbation Equations (APE-4) are solved in the timedomain. Results of the method's application for two aerofoil benchmark cases, with both sharp and blunt trailing edges are presented. In each case, the importance of individual linear and non-linear noise sources was investigated. Several new key features related to the unsteady implementation of the method were tested and brought into the equation. Encouraging results have been obtained for benchmark test cases using the new technique which is believed to be potentially applicable to other airframe noise problems where both tonal and broadband parts are important.
\end{abstract}

Key words: broadband and tonal noise, aerofoil noise, synthetic turbulence, FRPM, blunt trailing edge, vortex shedding 


\section{Introduction}

Aerofoil noise, or the noise generated by scattering of hydrodynamic field in the turbulent boundary layer close to the wing trailing edge, has been a subject of investigation since 1970s [1,2]. In recent years, this classical problem has kept attracting attention [3-6] and despite the availability of several experimental databases [7-9], an understanding of trailing edge noise mechanisms leading to robust scaling laws is still lacking.

Numerical modelling of aerofoil noise based on unsteady computational fluid dynamics approaches such as Large Eddy Simulation (LES) or Direct Numerical Simulation (DNS) came into practice in 2000 [10,11]. Since then, there have been approaches of various validity and complexity used for modelling the unresolved near-wall turbulence or directly resolving this for low Reynolds number flows [4,12-16]. For acoustic modelling, there has also been a range of formulations of various complexity used starting from Ffowcs Williams-Hawkings (1969) [17] and Amiet's theory (1976) [18] to solving the Acoustic Perturbation Equations (APE) [19] and performing direct noise computations [20,21].

A serious limitation of using LES for trailing edge noise modelling is their restriction to relatively low Reynolds numbers due to prohibitively high computational cost of resolving the boundary layer turbulence. This limitation has resulted in a very little use of LES in support of existing experimental aerofoil noise campaigns or industrial design processes where the computational cost is further increased due to the geometrical complexity. Therefore, attention turned to methods with a fast turnaround time, such as Reynolds-Averaged Navier-Stokes (RANS) simulations that evolved through 1990s and by the end of the decade were extensively used to obtain a time-averaged flow prediction for a wide variety of industrial problems with varying degrees of success. Despite its drawbacks in transition modelling and inability to accurately model the separation, RANS methods can provide a quick prediction for high Reynolds number flows typical to many industrial problems and therefore, these tools remain commonly used to the present day. Compared to LES the validity of acoustic prediction schemes based on RANS strongly depends on the model calibration. This also applies to hybrid RANS/LES methods [22] where a calibrated transition from one scheme to another needs to be performed. 
In the context of trailing edge noise modelling, URANS simulations have been used to predict the tonal noise generated by a bluff body vortex generator attached to an aerofoil boundary close to the trailing edge [23]. Pure tonal noise prediction schemes based on URANS were applied for multi-blade configurations in turbo-machinery, for example, in application to fan noise [24] and turbine noise [25] modelling with a reasonable computational efficiency. However, the ability of such schemes to provide reliable tonal noise predictions through estimating an isolated vortex shedding characteristic is rather questionable.

For broadband noise predictions, the stochastic Fast Random Particle Mesh (FRPM) method was developed [26-29] which can predict sound generated by turbulent flows over a wide range of Reynolds numbers. The approach is based on using RANS flow solution to generate synthetic turbulence whose statistics that matches the RANS calculation. The synthetic turbulence fluctuations obtained are then, typically, substituted into the right-hand-side sources of some acoustic formulation, the same way as the LES fluctuations would be, to propagate the acoustic solution to the far field.

More recently, the FRPM method together with APE for sound propagation was used [30] for fast-turn-around time acoustic calculations in the framework of Altus solver that is a proprietary code of BAE Systems. The solver applies the FRPM method on a Cartesian grid with the flow field interpolated from the RANS calculation to generate the sound sources. The sources are then interpolated onto an unstructured grid of general complexity around a scattering body to solve a set of Acoustic Perturbation Equations (APE-4 formulation) [19] using a high-order Quadrature-Free Discontinuous Galerkin method and the ADER scheme for time integration [31]. This solver is further developed to be used in the current work for broadband and tonal noise predictions.

Importantly, unlike the LES-based noise prediction schemes [32], which automatically account for all types of noise sources in the flow solution, the original FRPM model can only simulate broadband fluctuations which are generated by the stochastic particles moving with the time-averaged RANS flow field. For example, the original FRPM model cannot include any unsteady flow features such as vortex shedding or pairing which would produce tones in the noise spectra. However, under the scale separation assumption between the highfrequency turbulence fluctuations and the low frequency tones typical of the 
URANS solution methods, the tones should also be possible to incorporate in the corresponding acoustic prediction scheme.

Recently, an attempt to combine the FRPM method with a URANS solution for improved broadband noise predictions called U-FRPM method was developed for a centrifugal fan noise problem [33]. However, the underlining acoustic formulation used in that work remains unclear. For example, the UFRPM model appears to be based on simply adding up squares of two far-field pressure amplitudes, one being the broadband signal from FRPM and the other is the tonal signal from a separate steady-state model, to obtain the final power spectral density amplitude at the far-field observer location. Thus, first of all, this approach requires two acoustic calculations of the sound propagation to the far field for a single flow case that may be expensive. Moreover, such simplified treatment does not only ignore any possible nonlinear source interaction but also neglects any acoustic interference of the different source components that are assumed to be uncorrelated at the far field despite sound propagation effects, which assumption needs to be verified.

The current work is devoted to developing a consistent modelling framework for combining the flow scales responsible for the broadband and tonal noise generation at the source level in the FRPM scheme and implementing it in an engineering code such as ALTUS. The article is organised as follows:

- In Section 2, the governing acoustic formulation based on the APE is presented. The FRPM method and the numerical setup based on the RANS $k-\omega S S T$ [34] model and the finite-element solution of Acoustic Perturbation Equations are briefly reviewed.

- In Section 3, basic numerical model verification results are presented. First, the RANS flow solutions for two benchmark trailing edge noise configurations with a sharp and a blunt trailing edge are demonstrated. Then, for verifying the numerical propagation solution, an analytical sound propagation test is considered where the current numerical solution is compared with theory.

- In Section 4, acoustic modelling results for two benchmark noise configurations, with and without the tonal noise component, are considered and validated in comparison with the experimental data. In each case, the relative importance of various linear and non-linear noise sources is investigated. 


\section{Governing Acoustic Formulation and Computational Method}

Following [19], the governing non-homogeneous Acoustic Perturbation Equations are considered which correspond to the vorticity- and entropy-less Linearised Euler Equations

$$
\begin{aligned}
& \frac{\partial p^{\prime}}{\partial t}+\frac{\partial}{\partial x_{j}}\left(c_{0}^{2} \rho_{0} u_{j}^{\prime}+p^{\prime} u_{0_{j}}\right)=0 \\
& \frac{\partial u_{i}^{\prime}}{\partial t}+\frac{\partial}{\partial x_{i}}\left(u_{0_{j}} u_{j}^{\prime}+\frac{p^{\prime}}{\rho_{0}}\right)=\boldsymbol{Q},
\end{aligned}
$$

where $p^{\prime}$ and $u^{\prime}$ denote pressure and velocity perturbations that the system solves for, $\rho_{0}$ and $u_{0}$ are the density and velocity of the time averaged mean flow with the local speed of sound $c_{0}$, and $\boldsymbol{Q}$ is the effective acoustic source vector. Time averaged quantities, namely pressure, density and velocity fields could be obtained from a separate calculation such as a RANS simulation. Subsequently, the acoustic source vector $\boldsymbol{Q}$ is calculated and provided to the system of Eqs. (1) and (2) at every time step of the simulation.

The acoustic sources are defined following the vortex sound theory model from [29] which includes the following three terms:

$$
\boldsymbol{Q}=-\left\{\boldsymbol{\omega}_{0} \times \boldsymbol{u}^{\prime}\right\}-\left\{\boldsymbol{\omega}^{\prime} \times \boldsymbol{u}_{0}\right\}-\left\{\boldsymbol{\omega}^{\prime} \times \boldsymbol{u}^{\prime}\right\},
$$

where $\boldsymbol{\omega}_{0}$ and $\boldsymbol{\omega}^{\prime}$ represent the mean flow vorticity vector and its fluctuation, respectively. The vorticity fields can be defined from the mean flow and fluctuating velocity component through the standard relationships, e.g. $\boldsymbol{\omega}^{\prime}=$ $\nabla \times \boldsymbol{u}^{\prime}$. The first two terms in Eq. (3) represent linear sources with respect to velocity and vorticity fluctuations, later referred to $\omega_{0} \times \boldsymbol{u}^{\prime}$ part as term I and to $\omega^{\prime} \times u_{0}$ part as term II, and the third one is quadratic in terms of the fluctuations. The third non-linear part $\boldsymbol{\omega}^{\prime} \times \boldsymbol{u}^{\prime}$ of the vortex source in Eq. (3) is thought to be smaller than the first two terms for low Mach number aerofoil flows at moderate angles of attack and by assumption is neglected. As discussed in [29] it is often the second, linear vorticity fluctuation term, $\boldsymbol{\omega}^{\prime} \times \boldsymbol{u}_{0}$ included while the rest of the sources are ignored. In the present work, all three source terms of Eq. (3) will be retained to verify their relative importance for the test cases considered.

In accordance with the original FRPM model, where various source descriptions could be implemented [29], the underlying part of the fluctuating 
solution field in Eq. (3) is obtained from synthetic turbulence generated using the method discussed below.

In order to obtain the fluctuating stream function component $\psi_{i}$ in Eq. (4) from which individual instantaneous variables of Eq. (3) are later determined, a random white-noise field $\mathcal{U}$ is represented by Lagrangian particle tracers which carry random numbers. Collectively, these stochastic particles have a zero mean property and are evenly seeded over the mesh in the region not occupied by the geometry. As the simulation progresses, these particles are convected with the local mean flow inside the defined FRPM source region. During run-time particles that leave the domain due to the mean flow convection are substituted by new particles at the in-flow boundary to preserve the same particle density in the flow domain. At every time step the acoustic sources are to be evaluated, a set of random values are interpolated onto the neighbouring Cartesian mesh node (as shown in Fig. 1). This represents an approximation to the convective whitenoise field. By using the area-weighting kernel function that incorporates the statistics of the local mean flow solution, and applying the additional weighting with the amplitude $\hat{A}$ one can obtain the corresponding solenoidal velocity field as a required input for the acoustic sources.

$$
\psi_{i}(\boldsymbol{x}, t)=\int_{V_{s}^{n}} \hat{A} G\left(\boldsymbol{x}-\boldsymbol{x}^{\prime}\right) U_{i}\left(\boldsymbol{x}^{\prime}, t\right) d^{n} x^{\prime}
$$

In Eq. (4) $\hat{A}$ is the amplitude of the filter that is the function of the local kinetic energy, $n$ indicates the dimension of the problem, and $V_{s}^{n}$ is the considered source region. $G$ is the filter kernel.

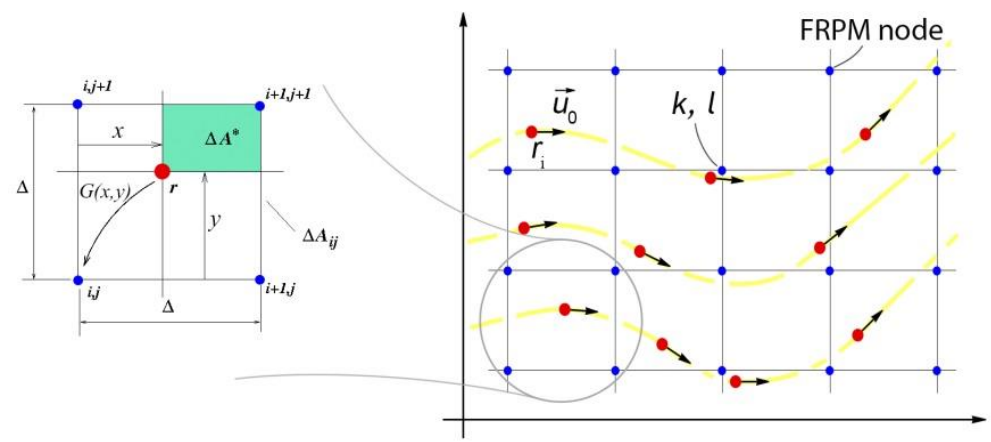

Fig. 1 - Random particles on mesh and area-weighting from particle to the grid point $\boldsymbol{G}(\boldsymbol{x}, \boldsymbol{y})=\Delta \boldsymbol{A}^{*} / \Delta \boldsymbol{A}_{\boldsymbol{i} \boldsymbol{j}}$, where $\Delta \boldsymbol{A}_{\boldsymbol{i j}}$ denotes the cell are related to lower left point $\boldsymbol{i}$. 
As shown in the previous literature on the FRPM method [26-28], for a Gaussian filter function $G(x)=\hat{A} \exp \left(-\frac{\pi}{2} \frac{x^{2}}{l^{2}}\right)$ the filter amplitude and width can be analytically expressed from the corresponding amplitude and scale of the autocorrelation function of the same velocity fluctuations, $R(x, d x)=$ $\overline{u^{\prime}(x+d x, t) u^{\prime}(x, t)}$ where the bar indicates time averaging. Similar to other RANS-based acoustic prediction schemes [35], the amplitude and the correlation scale can be further related to turbulence flow scales, as follows:

$$
l_{s}=\frac{c_{l}}{C_{\mu}} \frac{k^{\frac{1}{2}}}{\omega}
$$

In the above equation the local turbulent kinetic energy, $k$ and the specific dissipation, $\omega$ could be chosen as time-averaged or instantaneous values from URANS and the length scale, $l_{s}$ is expressed in terms of the $k-\omega$ model. The turbulent viscosity of the $k-\omega$ and $k-\epsilon$ models is related so that $\epsilon=$ $C_{\mu} k \omega$, where $C_{\mu}=0.09$ and $c_{l}$ is a calibration constant. In accordance with [3] its recommended value is in the range of $0.5-0.75$. In this work we use a fixed value for all calculations that is 0.72 . In accordance with [28], the scaling amplitude $\hat{A}$ of the filter function is linked to the turbulent kinetic energy via the relationship:

$$
\hat{A}=\sqrt{\frac{4}{3 \pi}} k^{\frac{1}{2}}
$$

Notably, there is a "frozen turbulence" assumption implied in the FRPM model, meaning that the random field is frozen in time and simply convects along the mean flow path without any de-correlation in time. Furthermore, the FRPM model is inherently steady, hence, cannot account for the unsteady effects such as vortex shedding, hence, the resulting acoustic model is not suitable for tonal noise. To account for the tonal noise sources with FRPM model, the present work uses the idea of scale separation and considers of a total velocity fluctuation consisting of the two parts:

$$
\boldsymbol{u}^{\prime}=\boldsymbol{u}_{f}^{\prime}+\boldsymbol{u}_{L}^{\prime}
$$


where $u_{f}^{\prime}$ is the 'fine-scale' fluctuating velocity component obtained from stochastic particles in accordance with the original FRPM scheme and $u_{L}^{\prime}$ is the 'large-scale' fluctuating velocity component. The latter can be obtained from a vortex-shedding resolving unsteady RANS (URANS) solution as a fluctuation of the time mean:

$$
\boldsymbol{u}_{L}^{\prime}=\boldsymbol{u}_{U R A N S}(t)-\boldsymbol{u}_{0}
$$

where $\boldsymbol{u}_{\text {URANS }}(t)$ is the unsteady URANS flow solution and $\overline{\boldsymbol{u}_{U R A N S}(t)}=\boldsymbol{u}_{0}$ is its corresponding time-average field.

From the resulting velocity fluctuation field Eq. (7), the fluctuating vorticity is obtained via a numerical differentiation as previously mentioned that by definition will also incorporate the $u_{L}^{\prime}$ term. The resulting data fields are then manipulated into the governing acoustic source equations, Eq. (3). For far-field sound propagation modelling, the APE equations with the source on the RHS are solved in a general conservation form:

$$
\frac{\partial \boldsymbol{U}(x, t)}{\partial t}+\frac{\partial \boldsymbol{F}_{j}}{\partial x_{j}}=\boldsymbol{Q}(\boldsymbol{x}, t)
$$

where $\boldsymbol{U}$ and $\boldsymbol{F}_{j}$ are the corresponding solution and flux vectors, j=1,2,3, and Einstein summation over the repeated index is implied.

For numerical computation with the Discontinuous Galerkin scheme [36] [37], the flow solution, the flux vectors and the sources are expanded in terms of the finite-element basis functions $\phi_{k}\left(x_{i}\right)$, e.g.

$$
\begin{aligned}
U(x, t) & =\phi_{k}(x) U_{k}(t) \\
F_{j}(x, t) & =\phi_{k}(x) F_{j_{k}}(t) \\
\mathcal{Q}(x, t) & =\phi_{k}(x) \mathcal{Q}_{k}(t)
\end{aligned}
$$

Following the standard weak solution procedure, the governing equations (9) are multiplied by the test function $\phi_{i}$, which are up to the $6^{\text {th }}$ order of approximation in this work, integrated over the volume with applying integration by parts and the divergence theorem. This leads to a system of ordinary differential equations for unknown time amplitudes $U_{k}$

$$
M_{k} \frac{\partial U_{k}}{\partial t}+\int_{\Gamma} \phi_{i} \phi_{k} F_{j_{k}} n_{i} d S-\int_{V} F_{j_{k}} \frac{\partial \varphi_{i}}{\partial x_{j}} \phi_{k} d V=\int_{V} \phi_{i} \phi_{k} \mathcal{Q}_{k} d V
$$


where the mass matrix is

$$
M_{k}=\int_{V} \phi_{i} \phi_{k} d V
$$

Due to linearity of the fluxes $F_{j}$ with respect to the acoustic variable the corresponding Jacobian matrix is pre-computed using the Quadrature-Free method. A RANS mean flow solution is mapped to the acoustic grid for the purpose of providing local density, pressure and velocity information. The flux reconstruction at the faces of control elements is achieved using the Roe fluxsplitting scheme. The equations are integrated in time using the $4^{\text {th }}$ order ADER method [31] of Titarev and Toro. All of these features are available in the framework of the Altus solver which is used in the present work.

For the 2D aerofoil profiles considered here, the computational domain for solving the acoustic propagation problem is covered by a triangular prism grid including the far-field "numerical microphone" location. The prismatic layer has one element in the span-wise direction that is the homogeneous direction of the problem. A symmetry plane condition is used in the span-wise direction and far-field boundary conditions are imposed at all other open-domain boundaries. At the aerofoil boundary, a no-slip wall ghost point boundary condition is applied.
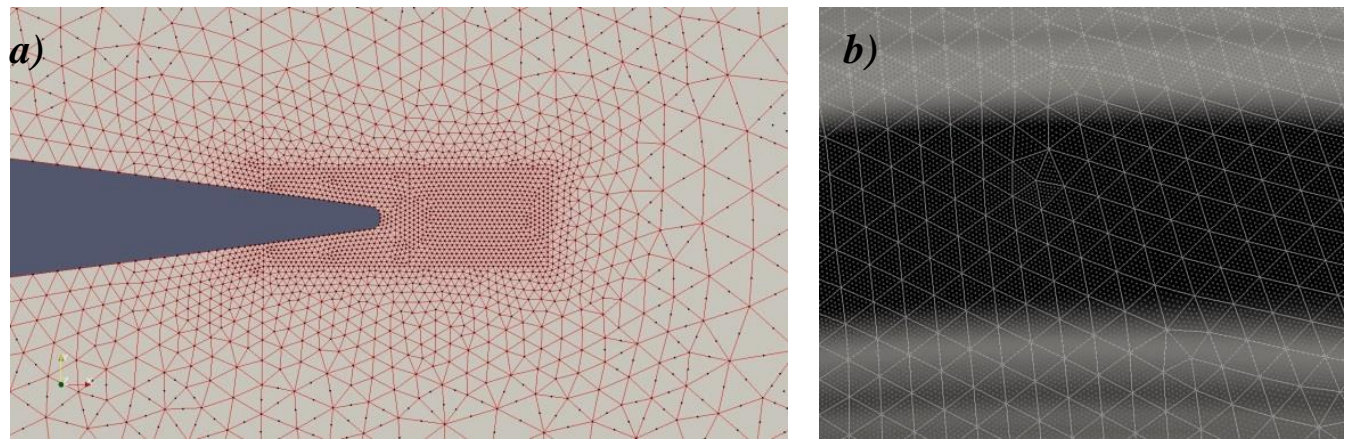

Fig. 2 - Computational acoustic grid in $x-y$ plane. (a) Grid elements in the vicinity of a trailing edge and $(b)$ the far-field showing high order elements over an instantaneous acoustic pressure wave.

For optimal computational efficiency of the current computational method, a variable order of the finite elements is used depending on the acoustic grid density, starting from the first-order elements in the finest grid region close 
to the aerofoil boundary and in the source region while using the $6^{\text {th }}$ order elements in the far-field region of a rapidly expanded grid, shown in

Fig. 2. Dots represent degrees of freedom.

For low Mach number flows of interest in the current publication, the acoustic propagation velocity is much larger compared to the hydrodynamic velocity. In application to the FRPM model this means that the acoustic time scale is very small as compared to the time scale required for the stochastic particle to travel any appreciable distance, in particular to traverse between the FRPM Cartesian grid cells. The same applies to the vortex shedding effect which scales with the local mean flow velocity rather than sound speed, allowing the URANS solution to march in time with a very large time step as compared to the acoustic wave propagation solution. To exploit the difference in the time scales, following [30], further computational savings are achieved by keeping the time step of the effective noise source computation an order of magnitude, 10 times larger in this case, in comparison with the acoustic propagation time step and using a linear interpolation to obtain the acoustic source distribution at the intermediate time steps. Following this procedure, the acoustic source generation part of the model takes only a fraction of cost of the entire model run time, most of which is spent on computing the acoustic wave propagation. In general, for the problems considered in this paper the computational wall clock times are about 48 hours per case including the spin-out time and the time required for the statistical solution post-processing on a small cluster of 64 computational cores. The spin-out time is defined as the time period required to reach a statistical stationarity of the acoustic solution. In practical terms, the spin-out time takes several throughflow times of acoustic wave propagation across the domain.

\section{Application to aerofoil noise modelling}

\subsection{Description of test cases and flow solution validation}

First, the benchmark NACA 0012 aerofoil case with a sharp trailing edge and zero incidence angle of attack from the workshop on Benchmark problems for Airframe Noise Computations (BANC) [38] is considered. The aerofoil 
chord length is $0.4 \mathrm{~m}$ and the free-stream velocity is $56 \mathrm{~m} / \mathrm{s}$, which correspond to a Reynolds number of about $1.5 \times 10^{6}$ and a free-stream Mach number of 0.1664 . The CFD part of a problem is solved with a 2D RANS simulation using the $k-\omega$ SST turbulence model with the advection scheme implemented in the form:

$$
\varphi_{i p}=\varphi_{u p}+\beta \nabla \varphi \cdot \Delta \boldsymbol{r}
$$

where $\varphi_{u p}$ is the flux value at the upwind node, and $\boldsymbol{r}$ is the vector from the upwind node to the integration point (ip). When the blend factor, $\beta$ is equal to zero, the scheme is simply first order upwind. For our simulations a non-linear recipe for $\beta$ based on the boundedness principles proposed by Barth and Jesperson [39] is used within a framework of the ANSYS CFX Solver, making the advection scheme second order accurate in space. The algorithm used can be shown to be Total Variation Diminishing (TVD) when applied to a onedimensional flow problem. The implicit time iterations are performed by the means of under-relaxation scheme.

A C-type mesh with 216 grid points per side of the aerofoil was generated paying special attention to the wake resolution zone behind the trailing edge. The grid resolution in wall-normal units, $y+$ is of the order of 1 , the far-field domain boundaries are placed 25 chords from the aerofoil leading edge and the total count of grid elements is approximately $10^{5}$. The mesh is shown in Fig. 3a. In addition, the grid refinement was performed in the streamwise direction using twice as many points per side of the aerofoil to demonstrate that trailing edge velocity and turbulent kinetic energy profiles in Fig. 4 are not very sensitive to the aspect ratio of the near-wall elements in the RANS solution.

It is important to mention here that in the framework of FRPM, the source modelling consists of two parts. One part is the RANS solution and the other is the FRPM particle emulation with the use of the corresponding auxiliary stochastic particle grid. The auxiliary grid is made consistent with the RANS solution which defines the corresponding filter length scale and amplitude of the stochastic particle distribution function as well as the particle convection speed. Hence, for consistency of the source modelling in FRPM, it is important to establish a low sensitivity of the statistical parameters to the RANS grid resolution. 
Fig. $3 \mathrm{~b}$ shows the numerical solution for the Mach number distribution around the aerofoil and the location of the "numerical probe" at $1.0038 x / c$ from the leading edge. The latter location is typical of the trailing edge noise sources and this is where the experimental flow data from the Institute of Aerodynamics \& Gas Dynamics (IAG) at University of Stuttgart is also available for comparison with the modelling as provided in [38].
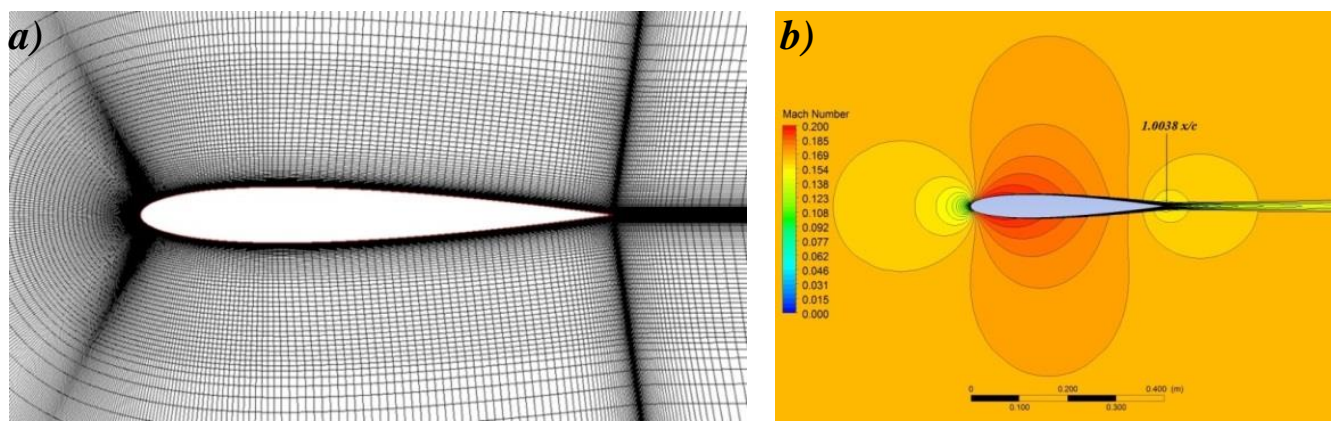

Fig. 3 - Computational grid in $x-y$ plane $(a)$ and the Mach number contours with the numerical probe location $(b)$

Fig. 4 compares the computed profiles of the mean flow velocity, turbulent kinetic energy, and the integral turbulence length scale, which characterise the convection speed, the amplitude and the filter length scale of the FRPM model for two RANS grid resolutions, with experiment at the 'numerical probe' location just downstream of the trailing edge. The mean flow profile is in an excellent agreement with experimental data including the inflection point being at $y / c \sim 0.035-0.04$ in the simulation, which is at the same location as reported in the BANC workshop for comparison [38]. The profile of the turbulent kinetic energy shows a good agreement with the experiment too with only minor excursions close to the centreline.

Notably, the definition of the integral turbulence scale length as applied in the experiment would require the determination of velocity auto-correlation function that is not available from the RANS simulation. Therefore, to be consistent with the RANS-based acoustic source modelling as discussed earlier, the turbulent scale is defined as the dimensional group combination involving the turbulent kinetic energy and energy dissipation rate with a calibration coefficient. See Eq. (5). In all calculations of the present paper the calibration 
length scale parameter, $c_{l}$ equal to 0.72 is used. This value shows a reasonable match with the experimental profile of the integral turbulence length scale apart from very small distances at the centreline.

a)

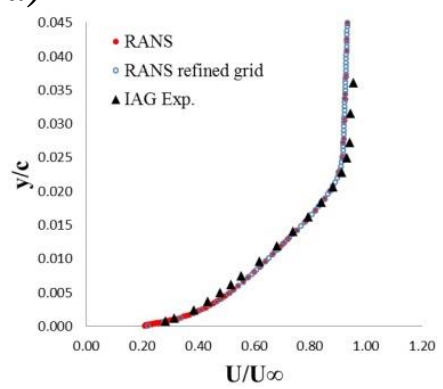

b)

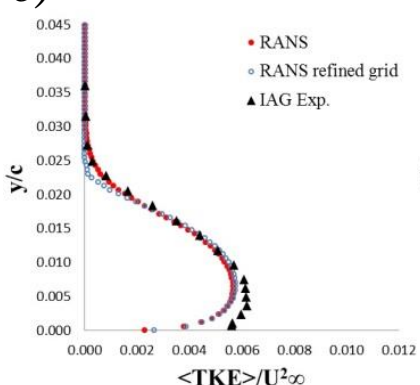

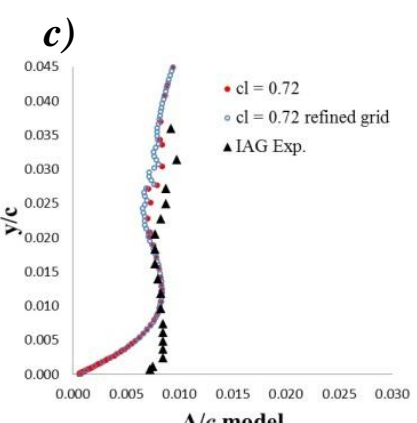

$\Lambda / c$ model

Fig. 4 Comparison of the RANS solutions on the standard grid and the grid that was refined in the stream-wise direction with the experiment downstream of the trailing edge: mean flow velocity (a), turbulent kinetic energy (b), and integral turbulence scale profile (c).

The second benchmark aerofoil noise problem considered in this work is the experiment of Brooks and Hodgson [43], for an aerofoil with a blunt trailing edge. The case chosen is that with the largest trailing edge thickness of 0.0025 $\mathrm{m}$ which exhibits pronounced tonal noise. The aerofoil used in the experiment was a NACA 0012 symmetrical aerofoil section with a chord length of 0.6096 $\mathrm{m}$ and a span of $0.46 \mathrm{~m}$ at zero incidence to the flow. The free-stream velocity is set to $U_{\infty}=69.5 \mathrm{~m} / \mathrm{s}$ and the corresponding Reynolds number based on the chord length is $2.77 \times 10^{6}$ with a free-stream Mach number equal to 0.2 . The blunt trailing edge leads to vortex shedding at $3 \mathrm{kHz}$ which corresponds to a Strouhal number of around 0.1 based on the free-stream velocity and the trailing edge thickness.

Similarly to the sharp trailing edge case, the problem is solved with the 2D $k-\omega$ SST turbulence model. In this case an unsteady RANS model is used to capture the vortex shedding. A second-order accurate scheme in space and time was applied for numerical solution on a C-type grid of approximately the same resolution in comparison with the sharp trailing edge case.

Two unsteady RANS simulations were performed for the blunt trailing edge problem. The first calculation was conducted for the same geometry as reported in the experiment while assuming a fully turbulent boundary layer 
condition on the aerofoil boundary. Compared to the experiment, where the transition to turbulence occurred due to the flow tripping on the sides of the aerofoil, the numerically predicted shedding frequency was approximately 2750 $\mathrm{Hz}$ more than $10 \%$ short of the experimental value.

Reproducing the correct transition to turbulence within the RANS model to replicate the aerofoil boundary condition in the experiment is very challenging and is likely to involve several calibration parameters of questionable validity since modelling of flow separation within the standard RANS framework could be questionable. Hence, no attempt to model the transition from a laminar to turbulent boundary layer is undertaken here. Instead, a simpler method to capture the correct shedding frequency is chosen for the second RANS simulation.

For the second RANS calculation, a slightly elongated aerofoil shape with the trailing edge thickness reduced by $20 \%$ is considered. This slight shape modification resulted in capturing the experimental shedding frequency of the 3 $\mathrm{kHz}$ numerically.

Fig. 5 compares the time-averaged URANS solution having the blunt trailing edge for pressure and skin friction coefficient $c_{p}$ and $c_{f}$ distributions with the experimental data of Gregory and O'Reilly [40] and Langley CFL3D RANS calculation. In comparison to the reference configuration of Brooks and Hodgson, the experiment of Gregory and O'Reilly together with Langley CFL3D solution correspond to the same aerofoil profile except for the sharp trailing edge, the same free-stream Mach number and a similar Reynolds number $\left(2.8 \times 10^{6}\right.$ vs $\left.2.77 \times 10^{6}\right)$. As can be seen the current URANS simulation is in very close agreement with data reported in the literature, where for this benchmark case the blunt trailing edge is only a small percentage of the aerofoil's thickness and therefore, the overall geometry could be deemed as almost identical. Notably, in the reference experiment Brooks and Hodgson reported a $c_{f}$ value of approximately 0.002 at the trailing edge, which is also in a good agreement with the current URANS simulation.

a)

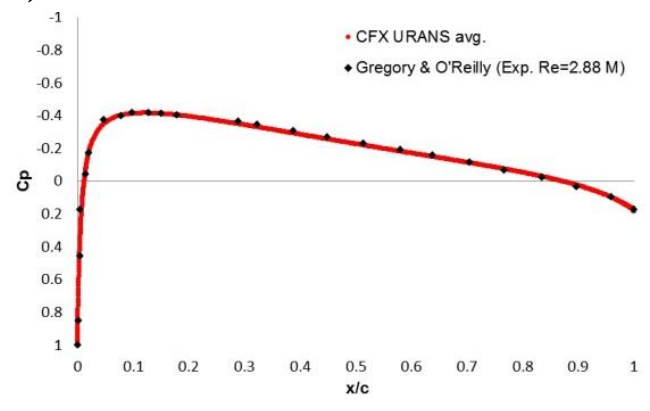

b)

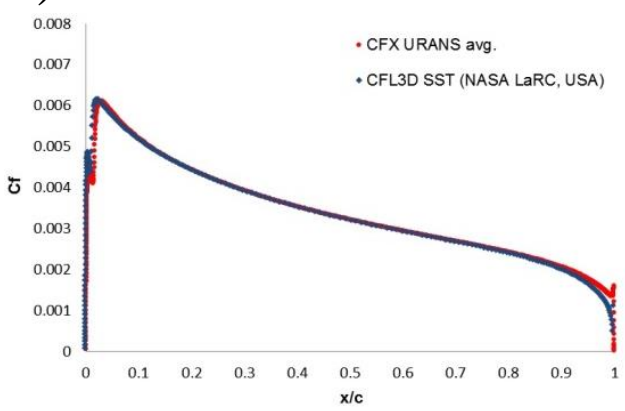


Fig. 5 - Comparison of the current RANS solutions with the available flow data for a similar NACA0012 aerofoil case from the literature: pressure (a) and skin friction coefficient distributions (b).

\subsection{Implementation of an acoustic source model for broadband and tonal noise simulations}

In order to minimise errors related to the filter scaling operation, the size of the Cartesian FRPM grid cell is kept much smaller than the smallest considered characteristic scale of the acoustic source. The latter scale is of the order of the turbulence length scale in the region of significant source amplitudes. In the current 2D simulations a cell size of 4 times as small as compared to the minimal value obtained by Eq. (5) is used. Where zero values are present in the source domain, a sufficiently small value thought to be of importance is picked as a reference scale. In our simulation this smallest scale within the source region was estimated to be in a range of $6 \times 10^{-4}$ with reference to the chord $C$, resulting in a cell size of $1.5 \times 10^{-4} \Delta / C$, where $\Delta$ is a Cartesian cell width. In addition, 10 stochastic particles per each Cartesian grid cell are specified. It was demonstrated that a sufficient number of particles are required within the FRPM domain [27] such that their area-weight contribution would achieve target root-means-square (RMS) values and therefore, yield a close approximation to Eq. (4). This ensures that parameters of the distribution of random particles vary slowly as compared to the convection scale of individual particles so that the particle contributions to the source are statistically converged. Importantly, in [27] converged statistics were obtained with an increase in the total number of particles beyond approximately 5 particles per cell.

Furthermore, in order to smoothly insert and eliminate particles without spurious noise amplification effects, a numerical decay function is built into the inlet and out zone of the FRPM domain which gradually attenuates the amplitude of the filter function.

When a tone is present in addition to the broadband fluctuations, it becomes an additional source of flow solution unsteadiness and consequently 
contributes to the noise. For source modelling, an unsteady flow solution obtained from URANS is mapped on to the same FRPM Cartesian grid where the synthetic turbulence flow component is generated. Fig. 6a shows a time averaged velocity magnitude field interpolated on to the FRPM grid which is required when evaluating $u_{L}^{\prime}$ at every CFD time step, shown in Fig. 6b. A sequence of CFD time steps that describe one complete period of shedding is selected. The parameters of interest that include turbulent kinetic energy, turbulent eddy frequency, mean and instantaneous URANS velocities along with the mesh information and CFD time step are recorded into a separate input file that are later used as an input for the acoustic solver.
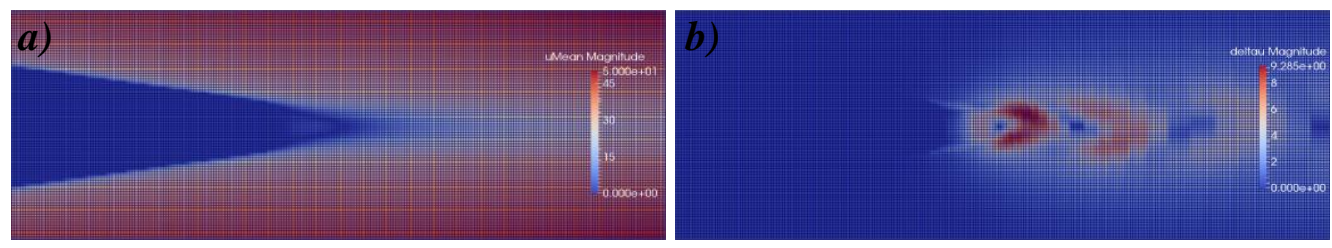

Fig. 6 - URANS solution provides an additional fluctuating velocity source for the blunt trailing-edge problem: mean velocity magnitude (a) and its fluctuation field mapped onto the FRPM Cartesian grid (b).

Fig. 7 shows the time history of cross-stream velocities in the wake zone normalised with a local mean kinetic energy at the numerical probe placed downstream of the trailing edge. Fig. 7 encompasses the reference $v^{\prime}$ FRPM velocity signal obtained from purely broadband stochastic sources as well as the modified $v^{\prime}+v_{L}^{\prime}$ velocity that incorporates the tonal noise harmonic. On the same plot, $v_{L}^{\prime}$ alone that is at the core of the tonal noise mechanism, shown with markers, represents large scale fluctuations which are a result of Eq. (6). Besides, the analytical harmonic function of $2750 \mathrm{~Hz}$ with an arbitrarily calibrated amplitude and phase is plotted to approximately fit the shedding frequency of the wake corresponding to the first URANS solution (Analytical).

It is interesting to observe how the stochastic part of the solution gets superimposed on the deterministic wave solution corresponding to the vortex shedding and results in the total signal which looks very much like a velocity fluctuation measured in a real experiment where tidal currents were recorded [41]. 


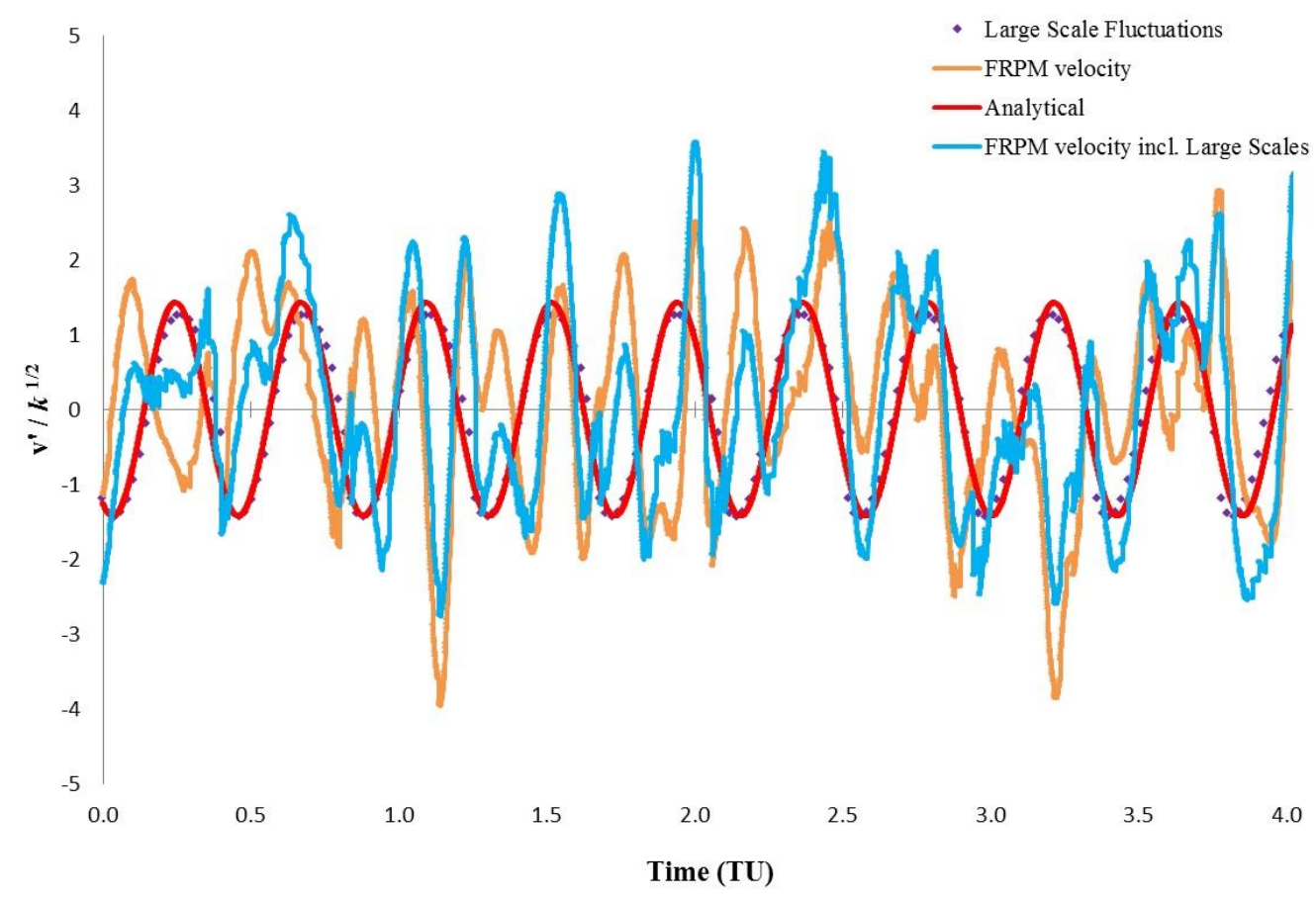

Fig. 7 - Time-domain behaviour of various velocity components behind the blunt trailing edge. The analytical function corresponding to the pure tonal velocity signal is included for comparison. $\sqrt{k}$ is a characteristic turbulent kinetic energy obtained from a 2D RANS solution. Time Units (TUs) are based on the free-stream velocity and the chord length, $\mathrm{TU}=\mathrm{C} / \mathrm{U}_{\infty}$.

In the current method based on the URANS solution there are two possibilities for realisation of turbulent kinetic energy and turbulent length scales on the FRPM grid. The choice is between using mean or instantaneous quantities for scaling the filter kernel in Eqs. (4-6). Our investigations with the current benchmark vortex shedding case show that acoustic predictions remain similar and consistent for both options. For the results discussed in Section 3.4 we used instantaneous fields for $k$ and $l$ directly obtained from parameters of the URANS simulation.

\subsection{Acoustic wave propagation solution}

Acoustic sources that incorporate both broadband and tonal mechanisms of noise generation described previously are evaluated at the FRPM grid nodes 
and then must be mapped to an acoustic grid for the wave propagation. However, due to the difference in mesh types where source interpolation from a Cartesian FRPM to an unstructured 2D prism acoustic grid takes place, the resolution of the latter in the source region should be roughly the same as of the FRPM grid for an improved accuracy of interpolation and accurate spatial representation of source terms.

Fig. 8a shows an example of the 2D grid in $X-Y$ plane used for acoustic propagation of the trailing-edge noise sources. The centre of the grid is slightly offset downstream from the aerofoil trailing edge location. This offset is used to improve the numerical efficiency of the far-field boundary conditions, as in this configuration, acoustic waves meeting the open far-field boundary would be normal to the boundary. Hence, possible numerical reflections are minimised. Fig. $8 \mathrm{~b}$ shows the snapshot of the acoustic pressure field in the vicinity of the aerofoil that is clearly of a dipolar nature along with the instantaneous representation of trailing edge acoustic sources.

a)

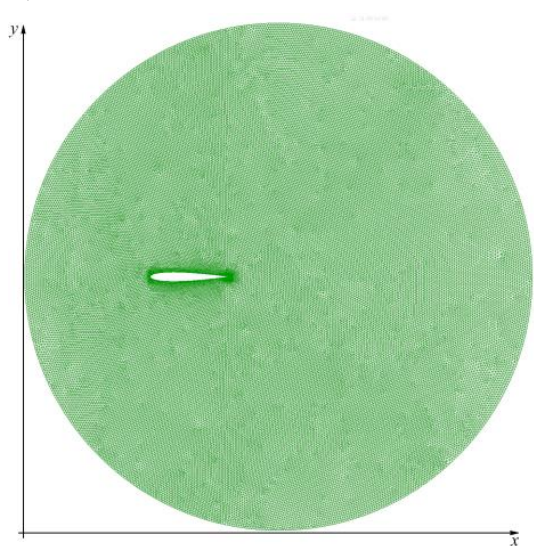

\section{b)}

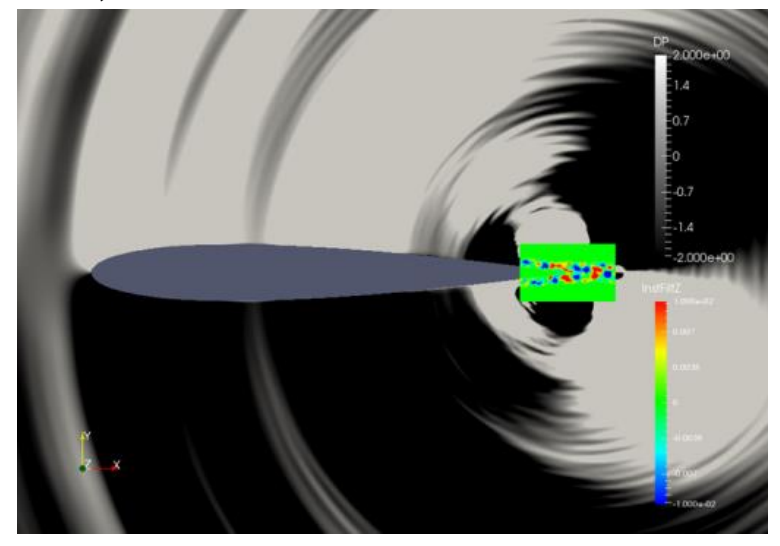

Fig. 8 -Acoustic wave propagation: an example of the computational grid used for solving sound propagation problem (a) and a typical snapshot of the acoustic pressure field with the hydrodynamic source region shown (b).

The acoustic grid is generated with the goal to resolve frequencies up to $10 \mathrm{kHz}$ with at least 2 elements of the order 6 per acoustic wavelength in the coarse grid region.

Having established a low sensitivity of the FRPM source model on the numerical grid resolution in section 3.1, to verify the performance of the 
numerical propagation model, the test problem which permits an analytical solution is considered. A cylindrical wave propagates from a point harmonic force at frequency $\Omega$, which corresponds to the fluctuating force, $f$ in the momentum equations so that $\boldsymbol{f}=-\delta(\boldsymbol{x}-\boldsymbol{y}) \boldsymbol{q}(\tau)$, where $\boldsymbol{q}(\tau)=\boldsymbol{A} \exp (i \Omega \tau), \boldsymbol{x}$ and $\boldsymbol{y}$ are the observer and the source coordinates, respectively, and $\tau$ is the time in the source reference frame. In accordance with [42], the resulting acoustic propagation problem is governed by the non-homogeneous linear wave equation as the following:

$$
\left(\nabla_{x}^{2}-\frac{1}{c_{0}^{2}} \frac{\partial^{2}}{\partial t^{2}}\right) p=\boldsymbol{Q}
$$

where $\boldsymbol{Q}=\boldsymbol{\nabla}_{\boldsymbol{x}} \cdot \boldsymbol{f}=-\boldsymbol{q}(\tau) \boldsymbol{\nabla}_{\boldsymbol{x}} \delta(\boldsymbol{x}-\boldsymbol{y})$ is the effective source as expressed through the fluctuating force.

Eq. (16) can be solved by in cylindrical coordinates $(r, \Theta)$ centred at the source with the appropriate radiation condition at the far field $(r \rightarrow+\infty)$. The resulting solution for the Fourier wave amplitude of the acoustic pressure wave [42] is given by

$$
p=-\frac{i}{4} \kappa \mathcal{H}_{1}^{(2)}(\kappa r) \frac{\boldsymbol{x}-\boldsymbol{y}}{r} \cdot \boldsymbol{A} \exp (i \Omega \tau)
$$

where $\kappa=\Omega / c_{0}$ and $\mathcal{H}_{1}^{(2)}(\kappa r)$ is the Hankel function of the $2^{\text {nd }}$ kind. At large distances from the source, $r \gg 1 / k$ the asymptotic solution is valid

$$
p(r, t)=\frac{1}{\sqrt{8 \pi}} \kappa^{\frac{1}{2}} e^{-i(\kappa r-\pi / 4)} \frac{x-y}{r^{3 / 2}} \cdot A \exp (i \Omega \tau),
$$

which at a $90^{\circ}$ observer angle leads to a scaling of the pressure amplitude with frequency so that $|p| \sim \kappa^{\frac{1}{2}} \sim \Omega^{\frac{1}{2}}$.

For comparison with the analytical solution, a suitable 2D acoustic grid is generated by removing the aerofoil shape from the grid shown in Fig. 8a.

For zero flow conditions, the acoustic propagation equations are solved with a localised source in the momentum equations which approximate the fluctuating point source $\delta(\boldsymbol{x}-\boldsymbol{y})$ by a Gaussian profile, $\delta^{\text {(Numerical) }}(\boldsymbol{x}-\boldsymbol{y})$

$$
\delta^{(\text {Numerical })}(\boldsymbol{x}-\boldsymbol{y})=e^{-\ln 2 \frac{|x-y|^{2}}{\sigma^{2}}}
$$


where the characteristic size of the numerical source distribution, $\sigma$, was taken to be around 4-5 grid elements. The latter choice was a compromise between the grid resolution required to capture the source function numerically and making sure that the source remains compact, e.g. $\sigma \ll 1 / \kappa$.

Fig. 9 shows the result of comparison between the calculations and the theory in terms of the far-field sound pressure amplitude when increasing the sound frequency, $\Omega$ while keeping all other test parameters the same. The observer location corresponds to a typical position of the far-field microphone in the trailing edge noise experiments at a $90^{\circ}$ observer angle to the chord. The amplitude of the numerical solution is Fourier transformed from the corresponding pressure signal. The amplitude of the analytical solution is calibrated so that it exactly matches the numerical solution at the lowest sound frequency corresponding to $2 \mathrm{kHz}$ and then scaled in accordance with theory so that $|p| \sim \Omega^{\frac{1}{2}}$.

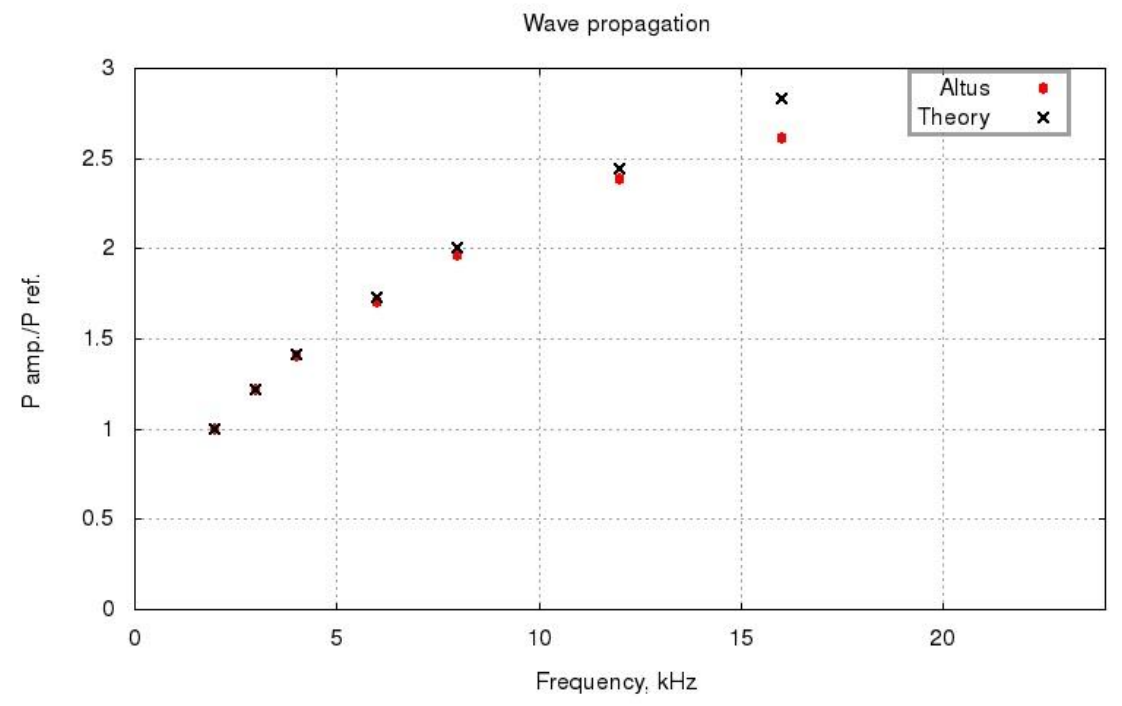

Fig. 9 - Comparison with the analytical solution for the acoustic wave propagation problem

The numerical solution is within 5\% from the analytical solution up to frequencies around $12 \mathrm{kHz}$, which demarcates the limit of the numerical 
resolution. Eventually, propagation errors become larger as one would expect beyond the frequency resolution limit of the grid where for $16 \mathrm{kHz}$ the average peak amplitude drops significantly in comparison to the expected value. However, even at the highest frequency considered, $16 \mathrm{kHz}$, the numerical solution deviates within $10 \%$ of the analytical solution meaning that in terms of the acoustic power on the Decibel scale, this is still within $1 \mathrm{~dB}$.

\subsection{Trailing edge noise results}

The acoustic data available for the sharp trailing edge experiment from the BANC workshop [38] provided by DLR corresponds to a microphone location at $1 \mathrm{~m}$ distance or 2.5 chord units from the aerofoil trailing edge and a $90^{\circ}$ observer angle. Fig. 10 compares the DLR data for the Sound Pressure Level $\left(\mathrm{SPL}=20 \log _{10} \mathrm{P}_{\text {avg }} / \mathrm{P}_{\text {ref }}\right) \quad 1 / 3$ octave band noise spectra with the current numerical predictions. The reference pressure level is taken as $20 \mu \mathrm{Pa}$. There are results for two implementations of the FRPM source model shown: one is the complete source model including the non-linear source in Eq. (3) and the other includes just the first two linear terms. Notably, for the current benchmark problem, the full source model including the nonlinear terms and the linear source model produce virtually the same noise spectra. This agreement is consistent with findings reported in [29] which discussed FRPM model results for broadband aerofoil noise predictions with the assumption that the nonlinear source terms are not important.

For comparison with the experiments which correspond to a finite span size, the amplitude correction has been applied to the noise predictions of the current 2D numerical models to account for 3D effects. Overall, the 2D source model implemented is thought to give a very close approximation to the sound sources found in the vicinity of the thin trailing edge where fluctuating quantities become quickly uncorrelated along the aerofoil's span. However, at low frequencies, the spanwise correlations which are not reproduced by URANS or the 2D source model may have an impact on far-field acoustics. Due to twodimensionality of the current FRPM-based predictions the current model is not applicable for low frequencies, estimated below $\sim 850 \mathrm{~Hz}$, where the span length of the aerofoil section becomes comparable to the acoustic wavelength. On the other hand, the noise behaviour at low frequencies requires a further 
investigation since the experimental data in the low frequency range is not available and accuracy of the empirical BPM model for these frequencies is questionable too. Following the 2D FRPM model framework by Ewert [3], the absolute levels of the numerical power spectra were adjusted by the same value to match the peak frequency of the experimental data.

The sound pressure levels were scaled with $10 n \log (M)$ for $2 \mathrm{D}$ to $3 \mathrm{D}$ correction proposed in the original work for the FRPM method, where $M$ is the free-stream Mach number and $n$ is a calibration parameter to match the required amplitude. In the present work the empirical calibration offset corresponds to $n$ $\sim 1.5$. This amplitude correction has been performed only once for the full source model corresponding to the sharp trailing edge experiment. The same amplitude calibration is then applied for all other models including the blunt trailing edge noise predictions considered in the end of this section. In essence, it can be argued that this amplitude correction can be agglomerated in the definition of the filter amplitude scaling based on the RANS flow solution as described in Eq. (6). Such agglomeration then leads to the RANS-based aerofoil noise prediction scheme to be dependent on two calibration parameters. Overall, the shape of the noise spectra is captured well including the roll-off at high frequencies which are within 3-4 dB from the experiment. Note that the empirical correction applied appears to be case sensitive and needs to be re-evaluated for a new class of trailing edge noise problems.

Fig. 11 compares contributions of different noise sources to the far-field noise spectra. Except for the low frequencies at which the current quasi-2D acoustic modelling is less valid, the source term II, $\boldsymbol{\omega}^{\prime} \times \boldsymbol{u}_{\mathbf{0}}$, remains dominant compared to all other terms for the sharp trailing edge noise case. Again, this finding is consistent with [29]. 


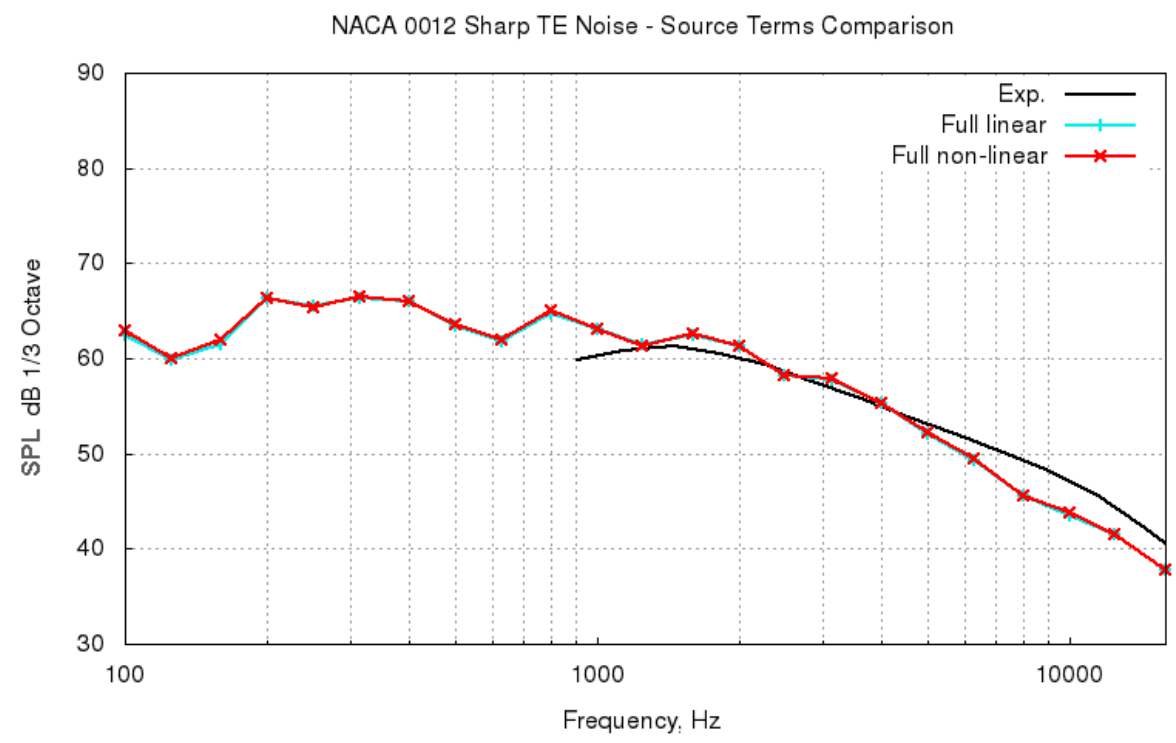

Fig. 10 - Comparison with the DLR experiment for the sharp trailing edge case: Sound Pressure Level (SPL) 1/3 octave band noise spectra predictions with and without including the non-linear sound source term.

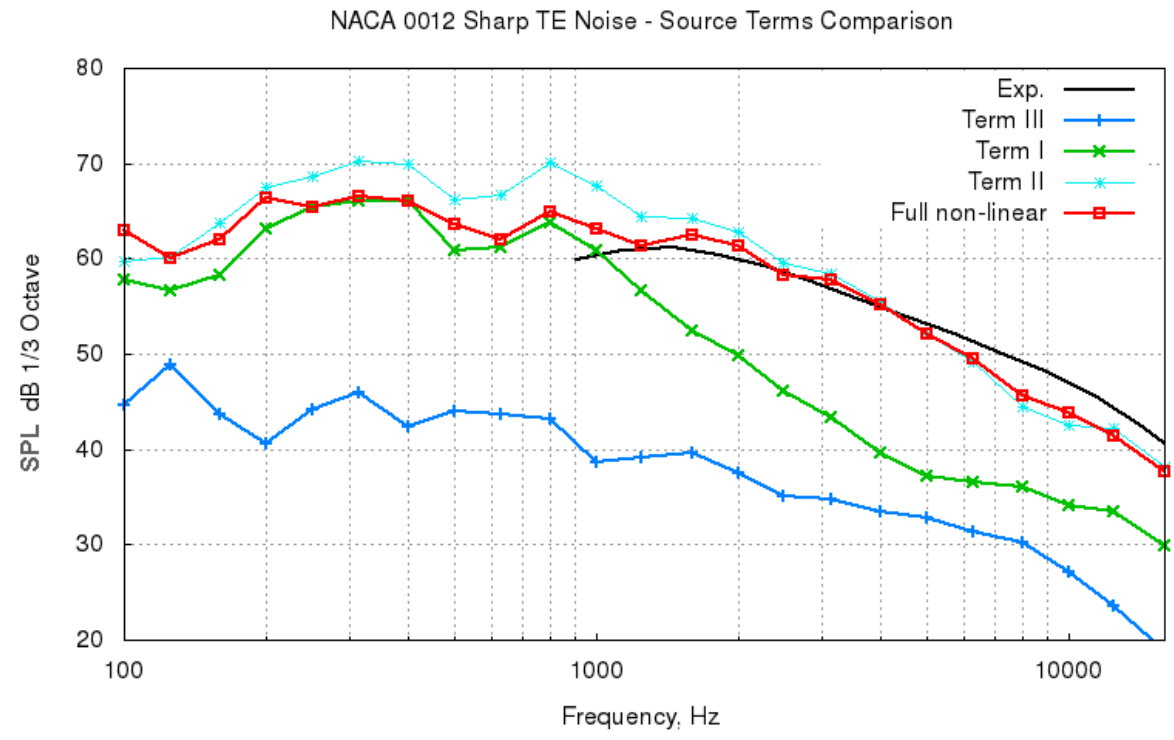

Fig. 11 - Sound spectra predicted by simulations employing individual noise source terms, Term I, II and III of Eq. (3) respectively and the full source 
model consisting of all source terms in comparison with the DLR spectra for the sharp trailing edge case.

For the blunt trailing edge experiment of Brooks \& Hodgson [43], the observer location is again at $90^{\circ}$ to the free stream and the distance to the farfield microphone corresponds to $L=1.222 \mathrm{~m}$ which is approximately 2 chords lengths. Fig. 12 shows the comparison of noise spectra predictions from various computations based on the first URANS calculation, which slightly under predicts the vortex shedding frequency of the experiment as discussed in Section 2, with the experimental data. Results of the empirical Brooks-Pope-Marcolini (BPM) solution from [7] are shown on the same plot for comparison. There are two solutions for noise spectra shown. The first solution is based on the standard FRPM formulation where the velocity source fluctuation includes only the stochastic turbulence component defined through the time-local URANS scale. The other solution is based on using the full velocity fluctuation including the tone given in Eq. (6). For comparison, the acoustic prediction corresponding to the isolated tonal part of the source, without the broadband part, which exhibits a secondary weak tone at $\sim 5 \mathrm{kHz}$, is shown in the same figure. It can be noticed that unlike either the pure broadband FRPM solution or the pure tonal noise solution, the prediction of the new unified approach includes both elements and is within $3 \mathrm{~dB}$ agreement from the experiment apart from some offset of the tone. As expected, the numerical tone prediction is shifted towards a lower frequency within 2-3 $\mathrm{kHz}$ range in accordance with the under prediction of the shedding frequency by the first URANS simulation. $3 \mathrm{~dB}$ is approximately the same error bar as demonstrated by the FRPM method implementation in the previous sharp trailing edge noise test.

To address the question how correlated are the broad band signal and the tonal noise at the far field, Fig. 13 compares the spectra prediction of the model that accounts both for the broadband and the tonal noise at the source level and the synthetic spectra obtained by simply adding squares of the acoustic pressure amplitudes of the pure broadband and the pure tonal noise signals in the far-field at the post-processing stage. The difference between the two spectra for the relevant frequency range is within $2 \mathrm{~dB}$ which means that within this error bar, which is within the accuracy of the current FRPM model, the two far-field signals are uncorrelated. 


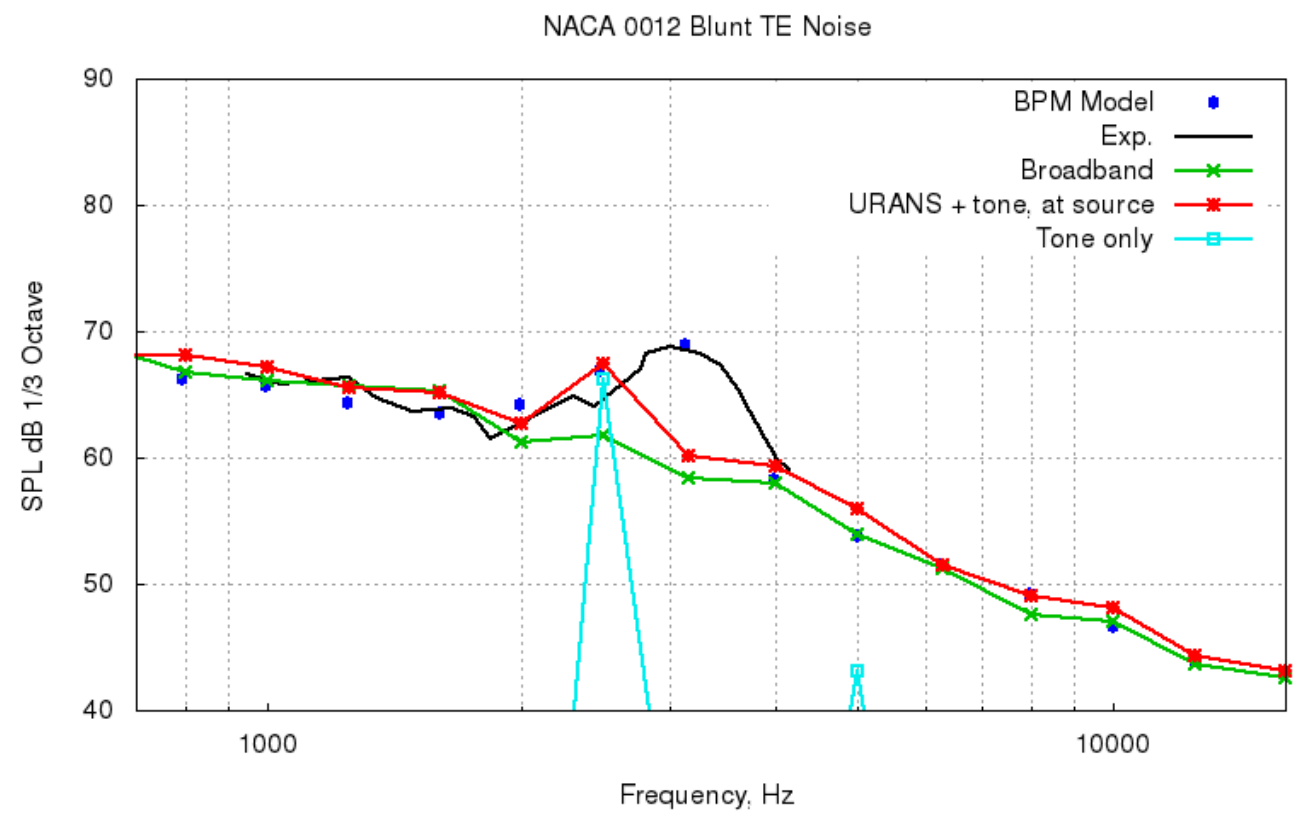

Fig. 12 - Comparison with experiment and the reference empirical model [7] for the blunt trailing edge case: Sound Pressure Level (SPL) 1/3 octave band noise spectra predictions based on the first URANS model with and without including the tonal noise source component and also for the pure tonal noise component. 


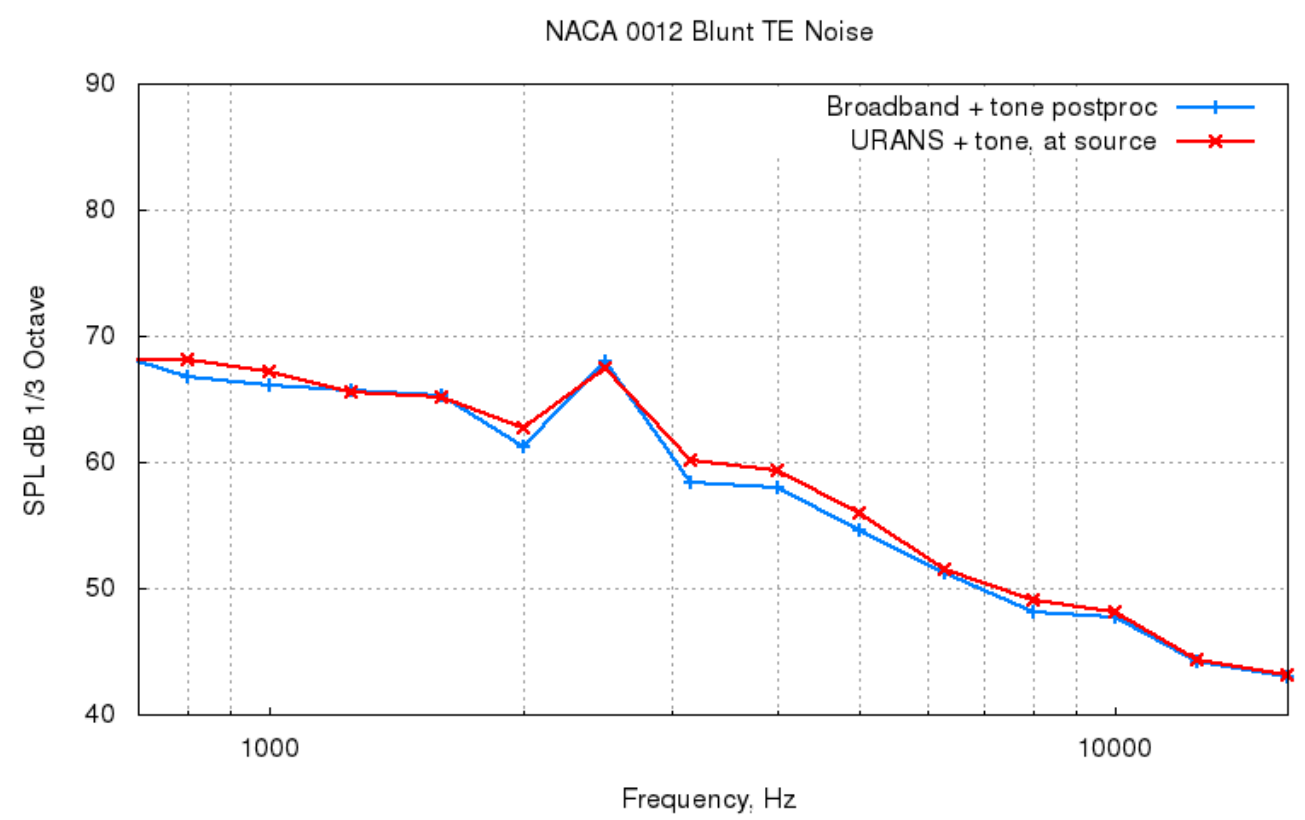

Fig. 13 - Sound Pressure Level (SPL) 1/3 octave band noise spectra comparison between the full term including the broadband and the tone at the source and the synthetic spectra obtained by simply adding squares of the acoustic pressure amplitudes of the pure broadband and the pure tonal noise signals in the far-field.

Fig. 14 shows the spectra predicted using the current URANS model with the tone included which are broken down into individual linear and non-linear source contributions in accordance with Eq. (3) : $\boldsymbol{\omega}_{0} \times \boldsymbol{u}^{\prime}$ (term I), $\boldsymbol{\omega}^{\prime} \times \boldsymbol{u}_{0}$ (term II), and $\boldsymbol{\omega}^{\prime} \times \boldsymbol{u}^{\prime}$ (term III) as well as the total spectra.

It can be observed that while term II remains dominant for the broadband part of the spectra as compared to the other 2 terms, term I is equally important in the region of the tonal peak. The importance of term I for the blunt trailing edge case is a clear distinction as compared to the sharp trailing edge flow, where the noise mechanism was purely broadband.

Interestingly, for the tonal peak, the noise contribution from source terms I and II are of a similar magnitude to the total signal. This means that the sound pressure powers produced by the two sources don't simply add up to produce the total as it would be the case if the acoustic source interference of these two source terms was negligible as it has been the case for the broadband and tonal noise 
components. Instead, the phase difference between the corresponding pressure signals produced by the two sources is close to $\pi / 2$. The latter is consistent with relating the tonal noise mechanism to a linear shear wave transport where velocity and vorticity fluctuations are offset in phase by $\pi / 2$. For the blunt trailing edge aerofoil case, the contribution of the non-linear term III remains insignificant in comparison with the linear sources as for the previous sharp trailing edge aerofoil problem. This further reconfirms that the interference of the tonal and broadband noise sources is not important in this case.

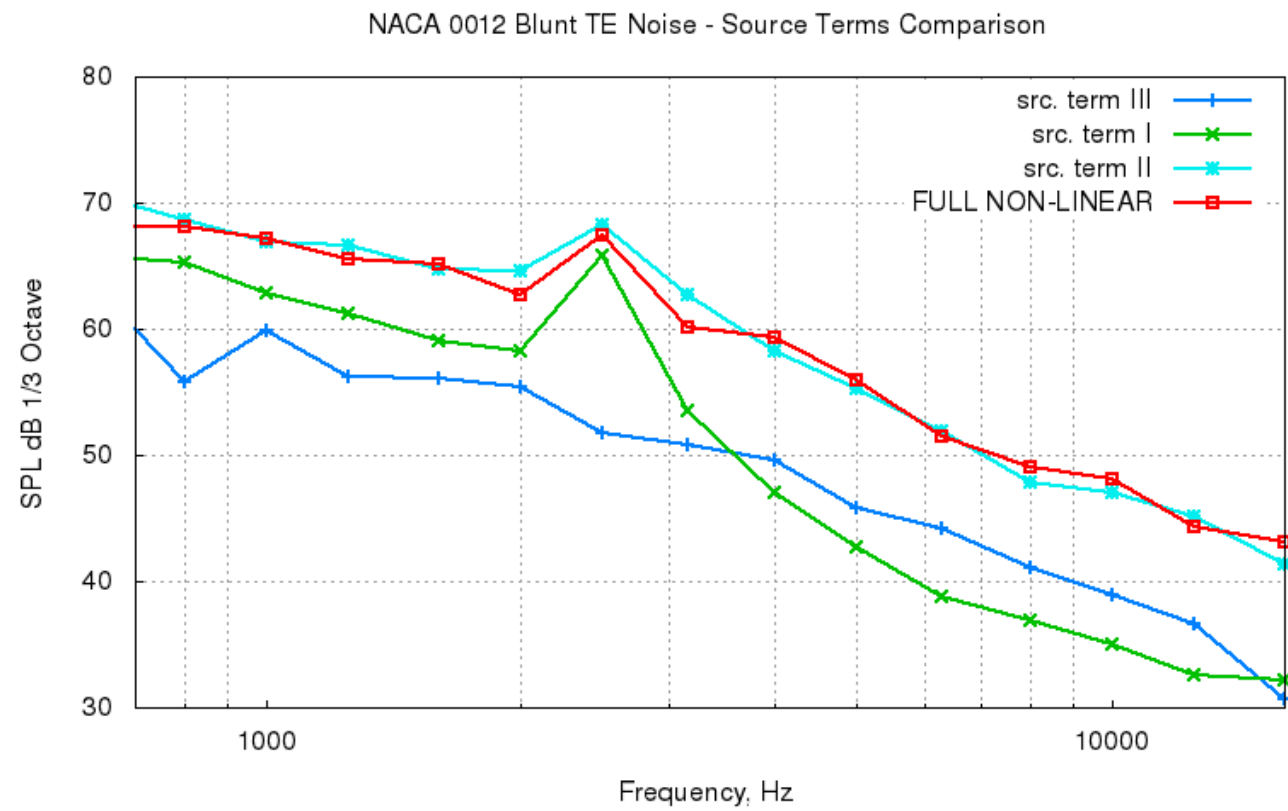

Fig. 14 - Sound level predicted by simulations employing individual noise source terms, Term I, II and III of Eq. (3) respectively and the full source model for the blunt trailing edge case

Fig. 15 shows the total noise spectra predictions obtained with the second URANS solution, which was fine-tuned in accordance with the description in Section 3.1 to reproduce the correct shedding frequency of the experiment. The acoustic predictions based on this second URANS solution are in excellent agreement with the experiment including both the broadband and the tonal part of the spectra. Overall, the current predictions are within 2-3 $\mathrm{dB}$ from the measurements. 
Importantly, except for the modified URANS solution, there was no other calibration used for obtaining the improved acoustic predictions as compared to the original FRPM model.

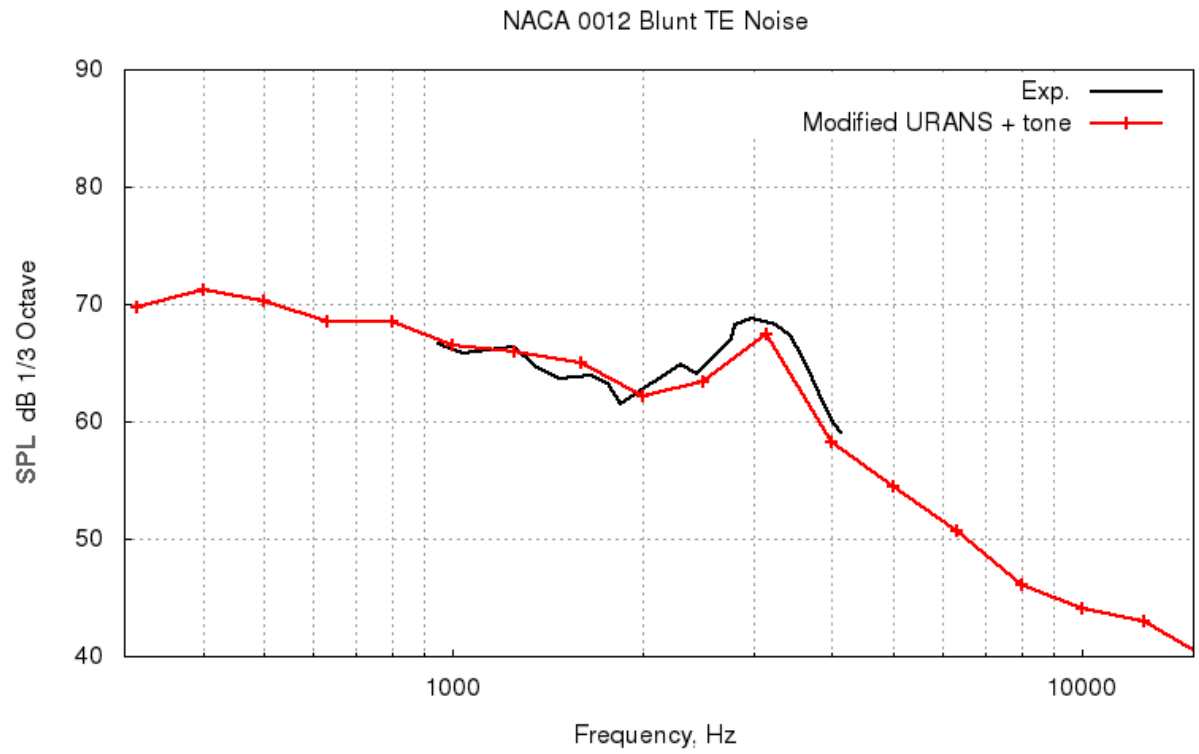

Fig. 15 - Comparison with experiment [43] for the blunt trailing edge case: Sound Pressure Level (SPL) 1/3 octave band noise spectra predictions based on the modified URANS flow solution.

\section{Conclusions}

For the aerofoil trailing-edge noise applications a Fast Random Particle Mesh (FRPM) method combined with solving the time-domain Acoustic Perturbation Equations is used in the framework of the BAE ALTUS solver. The simulations were performed for a 2D model setting which made them amenable to a 48 hour run time per case on 64 computational cores.

The original FRPM technique has been extended to include tonal noise sources based on the idea of scale separation by combining the large-scale flow solution available from Unsteady Reynolds-Averaged Navier-Stokes (URANS) with the fine-scale FRPM solution. Compared to the existing literature this 
modification allows for a unified treatment of the broadband and tonal noise sources at the source level, consistently accounting for source interference and possible nonlinear source interaction effects.

To validate the new model two benchmark aerofoil noise cases have been tested. The first case with a sharp trailing edge has come from the workshop on Benchmark problems for Airframe Noise Computations (BANC) having the near-filed flow and the far-field acoustic data available. The second case corresponds to the blunt trailing edge experiment conducted in 1980s by Brooks \& Hodgson for which only the far-field acoustic data is available. For both simulations, a NACA 0012 aerofoil section at zero incidence flow angle at a high Reynolds number of the order $2 \times 10^{6}$ has been used and for both cases, assuming fully turbulent boundary layer conditions, CFD solutions are obtained with encouraging agreement compared to the experimental flow data available in the literature.

For the blunt trailing edge case, a second URANS calculation is performed with a $20 \%$ reduced trailing edge thickness to exactly capture the vortex shedding frequency of the experiment. No modelling of the laminar/turbulent boundary layer transition occurring in the experiment was attempted.

For an analytical problem of a fluctuating point force specified in the governing acoustic equations and zero mean flow conditions, the accuracy of the current numerical wave propagation method for a grid resolution typical of the trailing edge noise problems of interest is verified in comparison with the theory and for the trailing edge noise predictions, the present simulations show an encouraging agreement $(2-3 \mathrm{~dB})$ with the experiment for both broadband and tonal noise. All model predictions are essentially based on the RANS simulations with just two calibration parameters: one for the correlation length scale and the other for the correlation amplitude.

The importance of including a separate tonal noise source in the original broadband FRPM model as well as having an appropriate flow model that captures the relevant tonal scale is investigated. By comparing the predictions of the new unified model with the synthetic spectra obtained by simply adding squares of the acoustic pressure amplitudes of the pure broadband and the pure tonal noise signals in the far-field, it is shown that the broadband and the tonal sources are virtually uncorrelated for the test case considered. However, not to 
mention its generality, the new unified approach is approximately two times computationally more efficient since the calculation of the synthetic spectra from the individual broadband and tonal signals requires 2 solutions of the far-field sound propagation problem.

Using the current modified FRPM model it is shown that while the linear source term II associated with the vorticity fluctuation is dominant for the broadband noise both linear terms I and II which involve the fluctuating vorticity and the fluctuating velocity can be significant for tonal noise. In the latter case the total far-field spectra is the result of acoustic interference of sources I and II which cannot be simply added up because of the phase difference. It is also confirmed that for the present benchmark cases the effect of the nonlinear source is negligible as compared to the linear sources.

Further work will include extending the new modelling framework based on FRPM to fully 3D flows and the use of LES data to inform the process of the acoustic source calibration.

\section{Acknowledgements}

The work has been supported by BAE Systems Ltd and the Engineering and Physical Sciences Research Council (EPSRC). One of the authors is grateful to the Royal Society of London for their continuing support. Computations were performed on the QM cluster 'Apocrita'. The authors are grateful to Dr Mark Allan for the initial introduction to the BAE Altus solver and fruitful discussions.

\section{References}

[1] J. E. Ffowcs-Williams and L. H. Hall, “Aerodynamic sound generation by turbulent flow in the vicinity of a scattering half plane," Journal of Flud Mechanics, vol. 40, no. 4, pp. 657-670, 1970.

[2] D. G. Crighton and F. G. Leppington, "On the scattering of aerodynamic noise," Journal of Fluid Mechanics, vol. 46, pp. 721-736, 1971.

[3] R. Ewert, C. Appel, J. Dierke and M. Herr, "RANS/CAA based prediction of NACA0012 broadband trailing edge noise and experimental validaton," in 15th AIAA/CEAS Aeroacoustics Conference (30th AIAA Aeroacoustics Conference), Miami, FL, 11-13 May 2011, 2009. 
[4] W. R. Wolf and S. K. Lele, "Trailing Edge Noise Predictions Using Compressible LES and Acoustic Analogy," in 17th AIAA/CEAS Aeroacoustic Conference, 05 - 08 June, Portland, Oregon, 2011.

[5] K. J. George and S. K. Lele, "Large Eddy Simulation of Airfoil Self-Noise at high Reynolds Number," in 22nd AIAA/CEAS Aeroacoustic Conference, 30 May - 1 June, Lyon, France, 2016.

[6] M. Herr, C. Appel, J. Dierke and R. Ewert, "Trailing-Edge Noise Data Quality Assessment for CAA Validation," in 16th AIAA/CEAS Aeroacoustics Conference, Stockholm, Sweden, 7-9 June, 2010.

[7] T. F. Brooks, D. S. Pope and M. A. Marcolini, "Airfoil Self-Noise and Prediction," NASA Reference Publication 1218, NASA, 1989.

[8] W. Devenport, R. A. Burdisso, H. Camargo, E. Crede, M. Remillieux, M. Rasnick and P. Van Seeters, "Aeroacoustic Testing of Wind Turbine Airfoils," National Renewable Energy Laboratory (NREL), Blacksburg, Virginia, 2010.

[9] M. Herr and M. Kamruzzaman, "Benchmarking of trailing-edge noise computatons: outcome of the BANC II Workshop," in 19th AIAA/CEAS Aeroacoustics Conference, Berlin, Germany, 27 May 2013.

[10] E. Manoha, B. Troff and P. Sagaut, "Trailing edge noise prediction using large-eddy simulation and acoustic analogy," AIAA J. , vol. 4, no. 38, pp. 575-583, 2000.

[11] M. Wang and P. Moin, "Computaton of Trailing-Edge Flow and Noise Using LargeEddy Simulation,” AIAA J., vol. 38, no. 12, pp. 2201-2209, 2000.

[12] M. Wang and P. Moin, "Dynamic wall modelling for large-eddy simulation of complex turbulent flows," Phys. Fluids, vol. 14, no. 7, pp. 2043-2051, 2002.

[13] A. L. Marsden, M. Wang, J. E. Dennis and P. Moin, "Trailing-edge noise reduction using derivative-free optimisation and large-eddy simulation," J. Fluid Mech., vol. 572, pp. 1336, 2007.

[14] R. D. Sandberg and N. D. Sandham, "Direct numerical simulation of turbulent flow past a trailing edge and the associated noise generation," J. Fluid Mech., vol. 596, pp. 353385,2008 . 
[15] L. E. Jones, R. D. Sandberg and N. D. Sandham, "Stability and receptivity characteristics of a laminar separation bubble on an aerofoil," J. Fluid Mech., vol. 648, pp. 257-296, 2010.

[16] V. A. Semiletov and S. A. Karabasov, "CABARET scheme for computational aero acoustics: extension to asynchronous time stepping and 3D flow modelling," International Journal of Aeroacoustics, vol. 13, no. 3-4, pp. 321-336, 2014.

[17] J. Ffowcs-Williams and D. Hawkings, "Sound generation by turbulence and surfaces in arbitrary motion," Philosophical Transactions of the Royal Society of London. Series A, Mathematical and Physical Sciences, vol. 1151, no. 264, pp. 321-342, 1969.

[18] R. K. Amiet, "Noise due to turbulent flow past a trailing edge," J. Sound and Vibration, vol. 47, no. 3, pp. 387-393, 1976.

[19] R. Ewert and W. Schröder, "Acoustic perturbation equations based on flow decomposition via source filtering," Journal of Computational Physics, no. 188, pp. 365$398,2003$.

[20] T. Le Garrec, X. Gloerfelt and C. Christophe, "Direct noise computation of trailing edge noise at high Reynolds numbers," in 14th AIAA/CEAS Aeroacoustics Conference, Vancouver, British Columbia, Canada, 5-7 May, 2008.

[21] T. Le Garrec and X. Gloerfelt, "trailing edge noise from an isolated airfoil at a high Reynolds number," in 15th AIAA/CEAS Aeroacoustics Conference, Miami, Florida, USA, 11-13 May, 2009.

[22] A. Garbaruk, M. Shur, M. Strelets and P. Spalart, "Jet Noise Computation Based on Enhanced DES Formulation Accelerating RANS-to-LES Transition in Free Shear Layers," in CEAA, Svetlogorsk, Russia, 22-26 Sept., 2014.

[23] B. A. Singer, K. S. Brentner, D. P. Lockard and G. M. Lilley, "Simulation of Acoustic Scattering from a Trailing Edge," J. Sound and Vibration, vol. 230, no. 3, pp. 541-560, 2000.

[24] S. M. Grace, D. L. Sondak, W. Eversman and M. J. Cannamela, "Hybrid Prediction of Fan Tonal Noise," AIAA, no. 2992, 2008.

[25] A. Serrano and G. Torres, "ESTIMATION OF TURBINE NOISE BENEFITS DUE TO ACOUSTICALLY TREATED OUTLET GUIDE VANES," in 11 th European 
Conference on Turbomachinery Fluid Dynamics \& Thermodynamics, Madrid, Spain, 2015, March 23-27.

[26] R. Ewert and R. Edmunds, "CAA Slat Noise Studies Applying Stochastic Sound Sources Based on Solenoidal Digital Filters," AIAA 2005-2862, 2005.

[27] R. Ewert, "RPM - the fast Random Particle-Mesh method to realize unsteady turbulent sound sources and velocity fields for CAA applications," in 13th AIAA/CEAS Aeroacoustics Conference (28th AIAA Aeroacoustics Conference), Rome, Italy, 21-23 May 2007, 2007.

[28] R. Ewert, "Broadband slat noise prediction based on CAA and stochastic sound sources from a fast random particle-mesh (RPM) method," Computers \& Fluids, vol. 37, pp. 369-387, 2008.

[29] R. Ewert, J. Dierke, J. Siebert, A. Neifeld, C. Appel, M. Siefert and O. Kornow, "CAA broadband noise prediction for aeroacoustic design," Journal of Sound and Vibration, vol. 330, pp. 4139-4160, 2011.

[30] M. Allan and O. Darbyshire, "Comparison of LES and Stochastic Source Generation Methods for Aero- and Hydro-Acoustic Design Guidance," in 20th AIAA/CEAS Aeroacoustics Conference, Atlanta, GA, 16-20 June 2014, 2014.

[31] V. A. Titarev and E. F. Toro, “ADER: Arbitrary high order Godunov approach,” Journal of Scientific Computing, no. 17, pp. 609-618, 2002.

[32] Y. Bae and Y. J. Moon, "A hybrid predictionmethod for low-subsonic turbulent flow noise," Computers and Fluids, vol. 39, pp. 1125-1135, 2010.

[33] S. Heo, C. Cheong and T. Kim, "Unsteady Fast Random Particle Mesh method for efficient prediction of tonal and broadband noises of a centrifugal fan unit," AIP Advances, vol. 5, no. 9, 2015.

[34] F. R. Menter, "Two-Equation Eddy-Viscosity Turbulence Models or Engineering Applications," AIAA J., vol. 32, no. 8, pp. 1598-1605, 1994.

[35] C. K. W. Tam and L. Auriault, “Jet Mixing Noise from Fine Scale Turbulence,” AIAA J., vol. 37, no. 2, pp. 145-153, 1999. 
[36] F. Bassi and S. Rebay, "High-Order Accurate Discontinuous Finite Element Solution of the 2D Euler Equations,” Journal of Computational Physics, vol. 138, pp. 251-285, 1997.

[37] H. L. Atkins and C.-W. Shu, "Quadrature-Free impementation of Discontinuous Galerkin method for hyperbolic equations," NASA Langley Research Center Contract 201594, Hampton, Virginia, USA, 1996.

[38] M. Herr, C. Bahr and M. Kamruzzaman, "Third Workshop on Benchmark Problems for Airframe Noise Computations (BANC III)," in 20th AIAA/CEAS Aeroacoustics Conference, Atlanta, Georgia, USA, 14-15 June, 2014.

[39] T. J. Barth and D. C. Jesperson, "The Design and Application of Upwind Schemes on Unstructured Meshes," in AIAA, 27th Aerospace Sciences Meeting, Reno, NV, USA, 912 Jan, 1989.

[40] N. Gregory and C. L. O'Reilly, "Low-Speed Aerodynamic Characteristics of NACA0012 Aerofoil Section, including the Effects of Upper-Surface Roughness Simulating Hoar Frost," Reports \& Memoranda No. 3726, London, 1970.

[41] I. A. Milne, R. N. Sharma, R. G. J. Flay and S. Bickerton, "Characteristics of the turbulence in the flow at a tidal stream power site," Phil Trans R Soc, 14 January 2013.

[42] D. G. Crighton, A. P. Dowling, J. E. Ffowcs Williams, M. Heckl and F. G. Leppington, "Modern Methods in Analytycal Acoustics," London: Springer-Verlag, 1996.

[43] T. F. Brooks and T. H. Hodgson, "TRAILING EDGE NOISE PREDICTION FROM MEASURED SURFACE PRESSURES,” Journal of Sound and Vibration, no. 78, pp. 69-117, 1981. 